\title{
Oral Cavity Verrucous Carcinoma
}

National Cancer Institute

\section{Source}

National Cancer Institute. Oral Cavity Verrucous Carcinoma. NCI Thesaurus. Code C8174.

A well differentiated squamous cell carcinoma arising from the oral cavity. It is an exophytic, warty, slow growing tumor, usually affecting older males. Chronic use of smokeless tobacco is associated with the development of verrucous carcinoma in the oral cavity. Five year disease-free survival rates between $80 \%$ and $90 \%$ have been reported in patients who underwent extensive surgical removal of the tumor. 